\title{
Acute Pulmonary Embolism with ST- Elevation Truly an Unusual Combination- but Still Exists! A Clinical Review of the Unusual Case
}

M Roelas ${ }^{1}$, M Halim², A Venkataraman ${ }^{2}$ and S Krishnamoorthy ${ }^{2 *}$

${ }^{1}$ FY1 in Cardiology, George Eliot Hospital, United Kingdom

${ }^{2}$ Consultant in Cardiology, George Eliot Hospital, United Kingdom

Submission: December 21, 2017; Published: February 09, 2018

*Corresponding author: S Krishnamoorthy, Consultant in Cardiology, George Eliot Hospital, College Road, Nuneaton, CV10 7DJ, United Kingdom,

Tel: 02476865 378; Fax: 02476 865704; Email: suresh.krishnamoorthy@geh.nhs.uk

\section{Abstract}

Acute pulmonary embolism (APE) is a cardiovascular emergency that without prompt clinical diagnosis and treatment may result in fatal outcomes. Indeed, the symptoms can be quite non-specific often causing clinical dilemma as well as potentially at risk of misdiagnosing on the initial presentation. More specifically, the electrocardiographic (ECG) manifestation of APE can be diverse from normal to sinus tachycardia with or without features of acute right heart strain. We present an unusual case of APE presented with the pattern of acute ST-elevation on the 12-lead ECG mimicking acute myocardial infarction; thereby discussing the clinical dilemma which arose with this case as well as all the potentially and relevant differential diagnosis for the presentation.

Keywords: ST- elevation; Acute pulmonary embolism; Acute myocardial infarction

Case Report

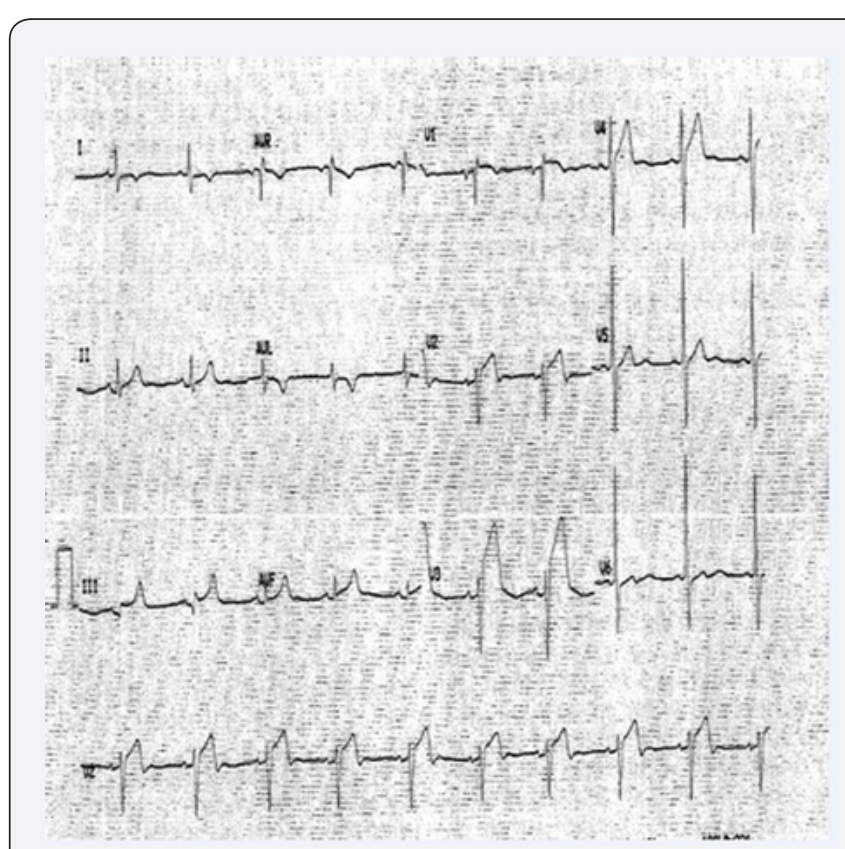

Figure 1: ECG whilst having chest pains with new ST elevation both in inferior and anterior leads.

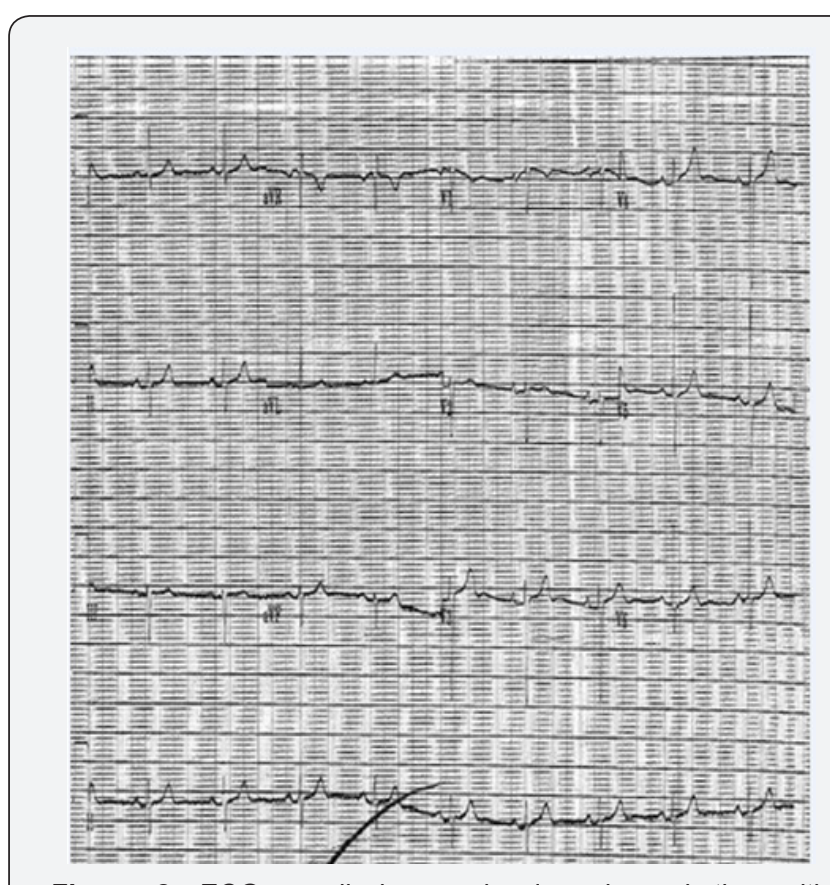

Figure 2: ECG on discharge showing sinus rhythm with complete resolution of ST-segment changes. 
63-yrs old female who was known to have treated hypertension admitted to a local district general hospital with an index episode of witnessed seizures whilst travelling in a bus. She does have co-existing diagnosis of right occipital meningioma confirmed 18-months ago, for which she was awaiting radiotherapy. Also, she has a previous history of unprovoked spontaneous APE, 1-year ago, with anti-cardiolipin antibody positive and has successfully completed 6-months course of warfarin. On arrival, she was postictal with altered consciousness with agitation, therefore was intubated and ventilated towards urgent CT head. This confirmed increase in the size of her meningioma with surrounding areas of oedema; however, without any acute bleeding or features suggestive of hydrocephalus. Post- ictal ECG showed sinus rhythm with no significant abnormalities. Her bloods were unremarkable including normal electrolytes and glucose. After successful respiratory wean and complete neurological recovery she was stepped down to a general medical ward 72-hrs later. Later that night, whilst in the toilet developed sudden onset central chest pains and sweatiness. She was found to be hypotensive with systolic $100 \mathrm{~mm}$ of $\mathrm{Hg}$ and hypoxic, despite clear lung fields. ECG revealed sinus bradycardia with ST elevation in antero-septal as well as inferior leads (Figure 1). She was given initial treatment for what clinically was thought to be a STEMI, including analgesics, antiplatelets (aspirin 300mg and ticagrelor $180 \mathrm{mg}$ ) and transferred to local tertiary cardiac centre for further evaluation. Her urgent coronary angiogram performed trans-radially, showed typical hypertensive tortuous vessels; but relatively otherwise normal coronaries (co-dominant system) with TIM-3 flow distally (Figure 2). The left ventriculogram showed the presence of good systolic function. However, her blood gas analysis showed resting hypoxia with p02 7.2KPa on room air and oxygen saturation of $86 \%$. She had complete resolution of ST-elevation with development of incomplete RBBB morphology in her QRS complexes. Her bloods revealed high sensitive Troponin-T of 149ng/L [normal reference $<15 \mathrm{ng} / \mathrm{L}]$ and D-Dimer of $8.52 \mathrm{mg} / \mathrm{L}$ Fibrinogen Equivalent, (FEU); [normal reference $<0.5 \mathrm{mg} / \mathrm{L} \mathrm{FEU].} \mathrm{Her} \mathrm{urgent} \mathrm{bedside}$ echocardiogram showed anon-dilated left ventricle with good systolic function without any regional wall motion abnormalities. Her right heart was mildly dilated with reduction in RV free wall contractility as well as with mild tricuspid regurgitation; however, with no signs of chronic volume or pressure overload. There was no obvious thrombus seen within the right heart and no pericardial effusion seen. Her subsequent CT-pulmonary angiogram confirmed (Figure 3a \& 3b) the presence of bilateral pulmonary embolism in her major pulmonary arteries without any evidence of infection or infarction or concurrent malignancies. The ultrasound venous Doppler of her legs revealed occluded right popliteal vein with thrombus. Subsequently following the neuro-surgical consult given her stable meningioma, she was started on anticoagulation with novel oral anticoagulant Rivaroxaban 15mg BD for 2 weeks and thereafter $20 \mathrm{mg}$ once daily planned for 6-months. Her subsequent out-patient bubble contrast echocardiography revealed intact inter-atrial septum without any presence of intracardiac shunts. Also, her cardiac
MRI revealed no evidence of myocardial infarction or fibrosis or infiltration in her ventricles. Notably, there were no features of late gadolinium enhancementor myocardial oedema observed on T1 \& T2-weighted imaging respectively. The right heart was nondilated with good systolic function and without any presence of intracardiac thrombus. Very importantly, investigations haven't identified the presence of occult malignancy as the underlying cause. As she remained clinically and haemodynamically well with normal saturations, she was discharged home and doing well on 3-month follow-up (Figure 4).

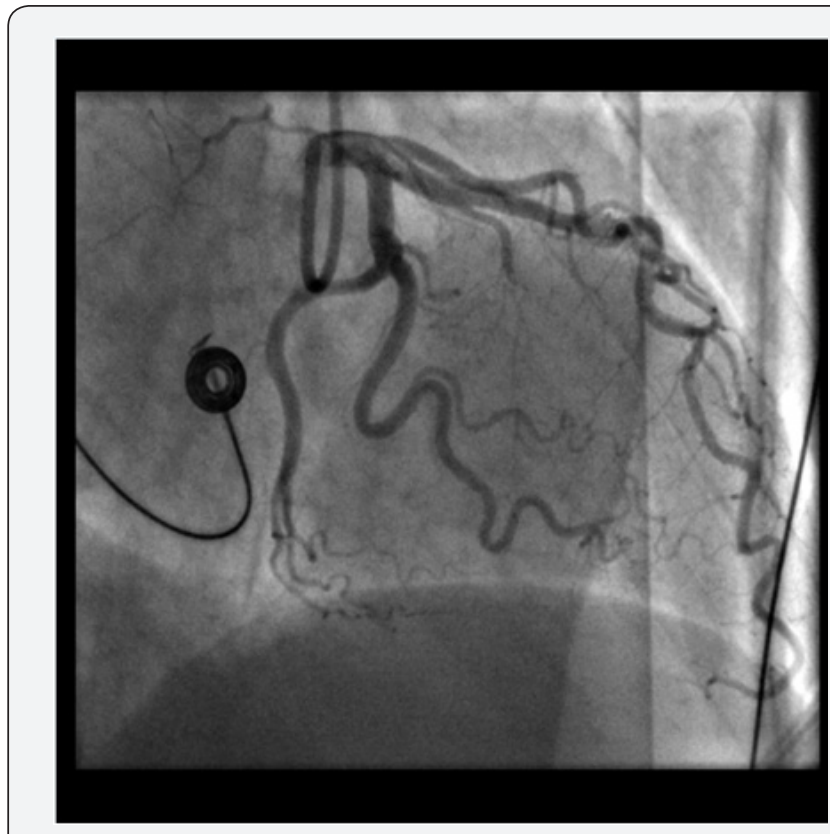

Figure 3a: Coronary angiogram of her left coronary system revealing normal tortuous coronaries.

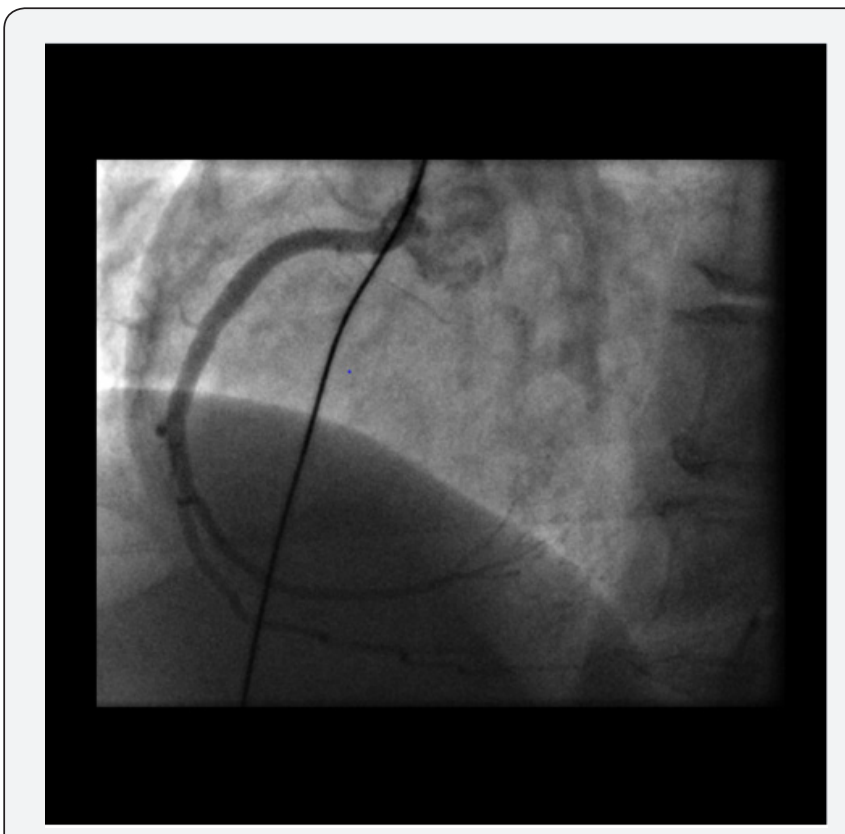

Figure 3b: Coronary angiogram of her right coronary system revealing normal tortuous coronaries. 


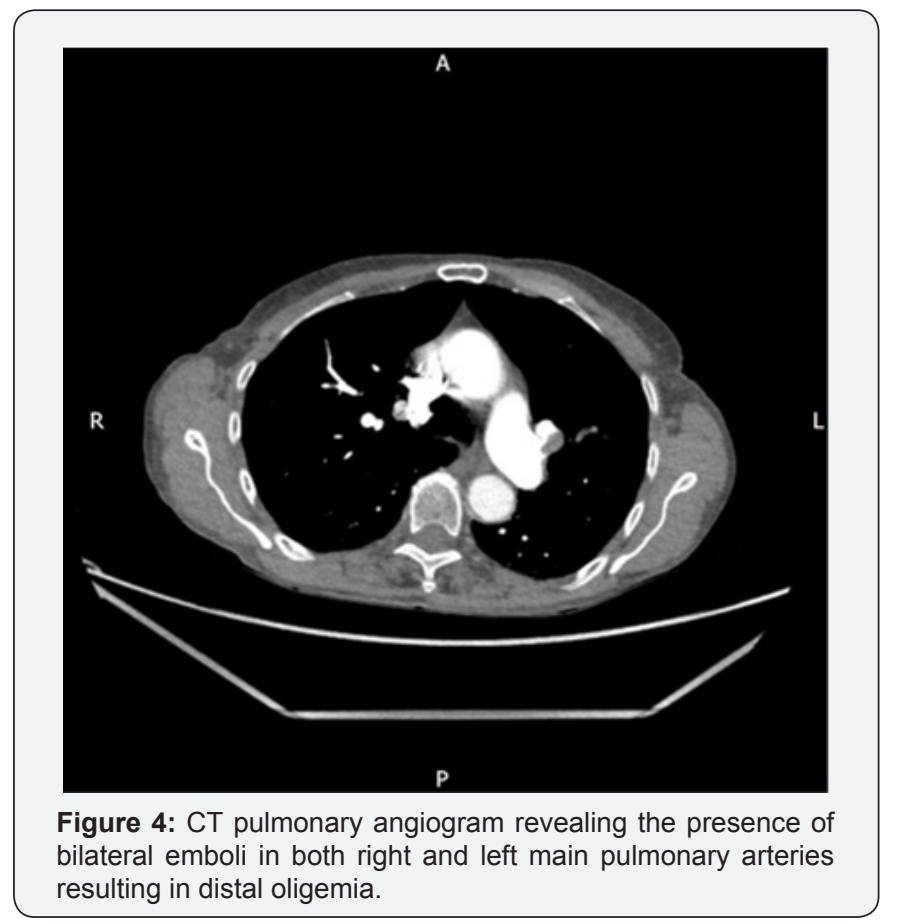

\section{Discussion}

It is rare and quite unusual for APE to present as acute ST-elevation on ECG. However, the initial clinical assessment favoured acute MI as primary diagnosis given her chest pains and an abnormal ECG, despite her slightly disproportionate degree of resting hypoxia with no chest signs.

On arrival, the differential diagnosis were:

\section{Acute ST-elevation myocardial infarction}

It is quite atypical and quite unusual to have two territory acute ischaemia (both right coronary artery and left anterior descending) given her ST elevation in inferior and anterior leads. Very rarely when it happens due to embolic phenomena or occlusion of left main stem or left main stem equivalent disease or a big wrap around left anterior descending artery, patients can be often both electrically and haemo dynamically unstable. Also, the concomitant respiratory distress, if present will be often due to acute pulmonary oedema with either typical or atypical clinical and radiological signs consistent with the above. Nevertheless, in this case the patient was hypoxic, notably with clear lungs on examination as well as chest X-ray, which was relatively unremarkable. Her coronary tree was essentially normal (without any evidence of plaque rupture or spontaneous coronary artery dissection or thrombus) with TIMI-3 flow in all the three major epi-cardial vessels on invasive coronary angiography. Albeit her elevated cardiac enzymes the left ventricular function both on ventriculography and echocardiogram was normal without any major regional wall motion abnormalities, makes the diagnosis of "acute myocardial infarction" very unlikely; even if this could be due to paradoxical embolism [1]. Her subsequent bubble contrast echocardiogram confirmed the presence of an intact inter-atrial septum without any other intra-cardiac shunts. However, her cardiac MRI revealed no evidence of myocardial infarction, fibrosis or infiltration. Also, it confirmed the presence of good LV systolic function without any regional wall motion abnormalities or valvular abnormalities.

\section{Tako-tsubo cardiomyopathy (transient apical ballooning syndrome, stress- induced cardiomyopathy, TSCM)}

TSCM represents a non-ischaemic form of acute heart failure, often preceded by physical or emotional trigger(s) or rarely in isolation. TSCM is primarily characterized by transient $\mathrm{LV}$ dysfunction and is associated with regional wall motion abnormalities on cardiac imaging. The clinical presentation of TSCM often mimics acute coronary syndrome (ACS) and more than three-quarters of patients with TSCM have abnormal levels of troponin as well as with ECG pattern(s) representing myocardial ischaemia [2]. Of note, acute ST elevation has been reported in one-third of patients with TSCM and often an urgent coronary angiogram is required to exclude any flow obstructive coronary lesions. Nearly $90 \%$ of patients will have no significant coronary artery disease accountable for the presentation. Though the precise patho physiological mechanisms behind the development of TSCM is not well elucidated; however, the commonly postulated mechanism include the acute catecholaminergic surge during the stress and the subsequent coronary spasm, micro vascular dysfunction, abnormal fatty acid metabolism, myocardial stunning, micro infarction and possible reperfusion injury [3].

Despite, classical TSCM was originally named after "octopus pot" as the left ventricle often has a characteristic shape due to transient apical ballooning with vigorously contracting basal segments during systole; however different atypical variants of TSCM without involvement of apical segments have been reported in the literature (i.e., inverted or reverse, mid-ventricular or localised). The modified mayo clinic criteria [4] is quite helpful in the diagnosis of TSCM with the presence of all four following features.

i. Transient hypo kinesis, a kinesis or dys-kinesis of left ventricular mid segments with or without involvement of apical segments -distinctively the extend of regional wall motion abnormalities observed will be beyond the distribution of single epicardial coronary vessel and with often a stressful trigger (but not always present).

ii. Absence of obstructive coronary disease or acute plaque rupture.

iii. New ECG abnormalities (either ST-elevation or T-wave changes) with modest elevation of troponin.

iv. Excluding the presence of phaechromocytoma or myocarditis. 
In postmenopausal women, Mugnai et al. [5] observed that the electrocardiographic changes with absence of abnormal Q waves and ST-elevation in V1 as well as with presence of ST depression in a VR were highly specific and positively predicts TSCM compared to anterior STEMI. Patients with TSCM, are often prone to heart failure, rarely cardiogenic shock as well as life threatening arrhythmias during the disease process. With the complete recovery of the transient LV dysfunction after the primary insult, the prognosis from TSCM is often thought to be quite favourable. However, recent evidences revealed 30-day and 1-year mortality as high as 5\% and $12 \%$ respectively with TSCM [6], thereby confirming its malignant potential as well as the need for close monitoring and management of these patients. Notably, in our case the patient's left systolic ventricular function was normal without any distinctive pattern of regional wall abnormalities both on ventriculogram, echocardiography as well as cardiac MRI excludes the diagnosis of TSCM. Of note, our patient's ECG revealed no ST-depression in a VR and the presence of ST-elevation in V1, which is again goes against the diagnosis of TSCM.

\section{Myopericarditis or perimyocarditis}

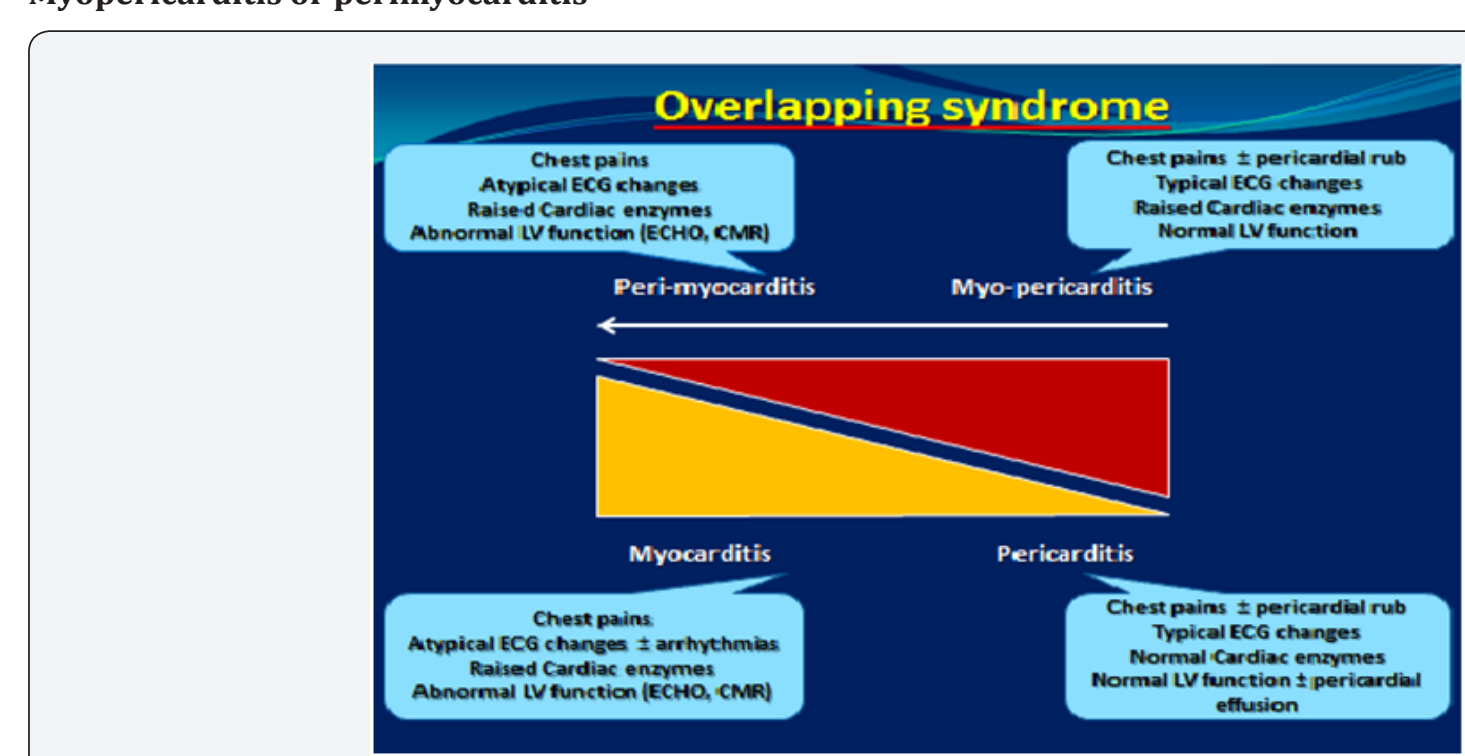

Figure 5: Schematic sketch of "myo-pericardial overlap inflammatory syndrome" with its individual components and their clinical assessment.

This overlap syndrome often represents the inflammatory process involving both pericardium and myocardium at variable degrees. Nevertheless, "myopericarditis" is primarily a pericardial disease with mild myocardial involvement (elevated cardiac biomarkers) and with normal cardiac function; in contrast "perimyocarditis" represents a primary myocarditic disease (elevated cardiac biomarkers) with some pericardial involvement and with abnormal cardiac function [7]. Despite the above, in routine clinical practice terms of "myopericarditis" and "perimyocarditis" are often used interchangeably as they tends to share similar aetiological agents causing the inflammatory insult Figure 5, illustrates the complex "myo-pericardial inflammatory overlap syndrome (MPOIS)" as well as those clinical parameters which helps in reaching the correct diagnosis; as both treatment and the prognosis from the condition(s) vary individually. The aetiology remains quite diverse and varies geographically; but commonly idiopathic (85\%), infectious (5\%) and immune mediated $(10 \%)$. Of note, cardiotrophic viruses (Coxsackie B, Adeno, CMV, EBV, Influenza, Varicella, Hepatitis B \& C, Parvo B19, HIV) have been more associated with MPIOS with their direct cytotoxic and cytolytic effects; thereby causing both acute and chronic inflammatory process causing cardiac dysfunction [7].

In acute phases, patients present with chest pains, fever, palpitations, myalgia, gastro intestinal symptoms and in chronic phases with more fatigue, breathlessness and reduced effort tolerance. In most patients, often a history of preceding or coexisting viral illness can be elicited. Depending on the degree of pericardial and or myocardial involvement, ECG changes varies from typical changes (diffuse concave ST-elevation and PR segment depression) to atypical forms (focal or segmental ST-elevation, T- wave changes and rarely arrhythmias) $[8,9]$. Abnormal cardiac biomarkers (Troponins, creatinine kinase- MB [CK-MB]) help in confirming myocardial involvement and often the levels correlate with the degree of inflammation. Notably in MPOIS patients, elevated troponin is not a predictor of adverse prognosis in contrast to ACS [10]. The conventional invasive coronary angiography often shows normal epicardial vessels. In myopericarditis, echocardiography usually shows normal cardiac function and rarely pericardial brightness and or pericardial effusion. However, in perimyocarditis, echocardiogram mostly demonstrates cardiac dysfunction (segmental or patchy or global) and similarly cardiac MRI often show typical changes ("patchy" sub-epicardial or "ring like" intramural late gadolinium enhancement on T1-weighted imaging and myocardial oedema on T2-weighted imaging) [11,12].

Given the lack of evidences with diverse aetiologies for the above condition, the treatment was primarily focussed on anti-inflammatory therapies in MPOIS patients with normal 
cardiac function. Precisely routine treatment with anti virals or corticosteroids are not recommended. Nevertheless, in patients with compromised cardiac function, conventional heart failure treatment is advisable with close monitoring on the recovery of cardiac function. Of note the prognosis associated with MPOIS appears quite favourable confirming complete recovery of cardiac function as well as both short- and long-term prognosis from Western registries $[8,10]$. In our clinical case, MPOIS is very unlikely had given her clinical presentation as well as with hypoxia. Notably, her ECG hasn't revealed any typical changes. Despite her abnormal troponin assay, her coronaries were normal and so does her LV systolic function. Similarly, both her echocardiogram and cardiac MRI confirmed the presence of normal cardiac function without any regional wall motion abnormalities without any typical changes as discussed above.

\section{Acute pulmonary embolism (APE)}

APE, is a common cardiovascular emergency condition, often refers to acute thrombo-embolic occlusion of pulmonary arterial tree causing acute hypoxia and hemodynamic instability. The mortality associated with APE in patients with hemodynamic insult can be as high as 30\% [13]. Often patients, presents with acute pleuritic chest pains, breathless, cough, haemoptysis, fever and rarely syncope [14]. A negative quantitative enzymelinked immune sorbent (ELISA) D-Dimer assay is highly valuable in excluding APE in patients with low or moderate pre-rest probability, given its high negative predictive value [15]. Early diagnosis can be achieved by ventilation-perfusion scintigraphy, pulmonary CT-angiography or conventional invasive pulmonary angiography [16]. Also, the judicious use of bedside echocardiography can be of immense clinical value in these emergent situations. Due to acute pressure overload from the PE, right heart may show evidence of RV dilatation, RV free wall hypo kinesis (Mc Connell sign) or RV dysfunction and rarely mobile right heart thrombus. Acute RV dysfunction with persistent arterial hypotension is highly predictive of adverse prognosis [17]. Given the lack of defined echo cardio graphic criteria as well as taking into consideration of the difficultly in evaluation of complex RV anatomy in conjunction with inter-observer variability, routine echo cardiographic examination is not recommended in non-high risk APE patients (who are haemo dynamically stable) [18]. Also, elevated plasma levels of both brain natriuretic peptide (BNP) or $\mathrm{N}$-terminal (NT)- pro BNP (marker of RV dysfunction) as well as Troponin (marker of myocardial injury) are highly predictive of mortality as well as poor outcomes $[19,20]$.

The clinical scenario in our patient fits very nicely with the diagnosis of acute pulmonary embolism given her atypical chest pains with diffuse ECG changes and resting hypoxia with no chest signs. Acute ST elevation has been reported in patients with acute pulmonary embolism [21-23]. Though the precise mechanism(s) behind those electrocardiographic changes have not been very well elucidated. Nevertheless, the below patho physiological changes may account for these transient changes: a. Acute right ventricular pressure overload in massive PE causing ischaemia with co-existing coronary spasm in both micro and macro-vasculature [24-26].

b. Excess catecholamine surge from either the haemo dynamic instability or hypoxia or chest pains with PE causing higher myocardial workload thereby ischaemia.

c. Low cardiac output state in massive PE and subsequent poor coronary filling with co-existent coronary stenosis.

d. Due to paradoxical embolus in the presence of intra-cardiac shunts like patent foramen ovale or atrial septal defect and coexistent venous clot giving rise to coronary embolus $[27,28]$.

Few case reports have revealed complete resolution of ST changes without any formation of pathological Q waves, following heparinisation in these patients with confirmed diagnosis of PE and STE $[29,30]$. Acute treatment of PE requires prompt and early confirmation of the diagnosis as well as appropriate hemodynamic and respiratory support with appropriate anticoagulation (parenteral i.e. unfractionated heparin or low molecular weight heparin or fond a parinux, vitamin-K antagonists i.e., warfarin, non-vitamin-K dependent new oral anticoagulants like direct thrombin inhibitors i.e., dabigatran or direct factor Xa inhibitors i.e., rivaroxaban or apixaban). However, urgent thrombolytic therapy is advocated only for high-risk and hemo dynamically unstable APE patients [31-33]. Other modalities of treatment like surgical embolectomy, percutaneous catheter based thrombectomy as well as venous filters are considered in patients with absolute contraindications to anticoagulation. Indeed, the duration of treatment with anticoagulation should be individualised guided by the risk factor for index presentation, VTE recurrences as well as their bleeding risk.

\section{Conclusion}

Pulmonary embolism can rarely present with ST-elevation on ECG. However, the responsible physician(s) needs to have high index of clinical suspicion and should often correlate the ECG findings appropriately in conjunction with the clinical presentation. If unusual on presentation all the possible and relevant differential diagnosis should be carefully explored. Adjunctive investigations invariably help in the early detection and confirmation of the diagnosis of acute pulmonary embolism as well as guide towards subsequent escalation of the treatment in these high-risk patients.

\section{References}

1. Krishnamoorthy S, Butt M, Lip GY (2010) Asymptomatic hypoxia in a young pregnant lady--unusual presentation of atrial septal defect. Int J Cardiol 143(2): E34-E36.

2. Sato H, Tateishi H, Uchida T (1990) Takotsubo-type cardiomyopathy due to multivessel spasm. In: Kodama K, Haze K, Hon M (Eds.), Clinical aspects of myocardial injury: from ischemia to heart failure (in Japanese). Kagakuhyouronsya, Tokyo, Japan, pp. 56-64.

3. Afonso L, Bachour K, Awad K, Sandidge G (2008) Takotsubo cardiomyopathy: pathogenetic insights and myocardial perfusion 
kinetics using myocardial contrast echocardiography. Eur J Echocardiogr 9(6): 849-854.

4. Kawai S, Kitabatake A, Tomoike H (2007) Guidelines for diagnosis of takotsubo (ampulla) cardiomyopathy. Circ J 71(6): 990-992.

5. Mugnai G, Pasqualin G, Benfari G, Bertagnolli L, Mugnai F, et al. (2015) Acute electrocardiographic differences between Takotsubo cardiomyopathy and anterior ST elevation myocardial infarction. J Electrocardiol 48(1): 79-85.

6. Stiermaier T, Thiele H, Eitel I (2016) Prognosis in patients with Takotsubo cardiomyopathy. Jacc Heart Fail 4(6): 519-520.

7. Imazio M, Trinchero R (2008) Myopericarditis: etiology, management, and prognosis. Int J Cardiol 127(1): 17-26.

8. Imazio M, Cecchi E, Demichelis B, Chinaglia A, Ierna S (2008) Myopericarditis versus viral or idiopathic acute pericarditis. Heart 94(4): 498-501.

9. Chan TC, Brady WJ, Pollack M (1999) Electrocardiographic manifestations: acute myopericarditis. J Emerg Med 17(5): 865-872.

10. Imazio M, Brucato A, Barbieri A, Ferroni F, Maestroni S, et al. (2013) Good prognosis for pericarditis with and without myocardial involvement: results from a multicenter, prospective cohort study. Circulation 128(1): 42-49.

11. Friedrich MG, Sechtem U, Schulz-Menger J, Holmvang G, Alakija P, et al. (2009) Cardiovascular magnetic resonance in myocarditis: a JACC white paper. J Am Coll Cardiol 53(17): 1475-1487.

12. Stensaeth KH, Fossum E, Hoffmann P, Mangschau A, Klow NE (2011) Clinical characteristics and role of early cardiac magnetic resonance imaging in patients with suspected ST-elevation myocardial infarction and normal coronary arteries. Int J Cardiovasc Imaging 27(3): 355365.

13. Wood KE (2002) Major pulmonary embolism: review of a pathophysiologic approach to the golden hour of hemodynamically significant pulmonary embolism. Chest 121(3): 877- 905.

14. Pollack CV, Schreiber D, Goldhaber SZ, Slattery D, Fanikos J, et al (2011) Clinical characteristics, management, and outcomes of patients diagnosed with acute pulmonary embolism in the emergency department: initial report of emperor (multicenter emergency medicine pulmonary embolism in the real world registry). J Am Coll Cardiol 57(6): 700-706.

15. Carrier M, Righini M, Djurabi RK, Huisman MV, Perrier A, et al. (2009) Vidas d-dimer in combination with clinical pre-test probability to rule out pulmonary embolism. A systematic review of management outcome studies. Thromb Haemost 101(5): 886-892.

16. Konstantinides SV, Torbicki A, Agnelli G, Danchin N, Fitzmaurice D, et al. (2014) Force for the diagnosis and management of acute pulmonary embolism of the European society of cardiology (ESC) ESC guidelines on the diagnosis and management of acute pulmonary embolism. Eur Heart J 35(43): 3033-3069.

17. Coutanceg G, Cauderliere E, Ehtishamj J, Hamonm M, Hamonm M (2011) The prognostic value of markers of right ventricular dysfunction in pulmonary embolism: A meta-Analysis. Crit Care 15(2): R103.
18. Roy PM, Colombet I, Durieux P, Chatellier G, Sors H, et al. (2005) Systematic review and meta-analysis of strategies for the diagnosis of suspected pulmonary embolism. BMJ 331(7511): 259.

19. Klok FA, Mos IC, Huisman MV (2008) Brain-type natriuretic peptide levels in the prediction of adverse outcome in patients with pulmonary embolism: a systematic review and meta-analysis. Am J Respir Crit Care Med 178(4): 425-430.

20. Jime'Nez D, Uresandi F, Otero R, Lobo JL, Monreal M, et al. (2009) Troponin-based risk stratification of patients with acute non-massive pulmonary embolism: systematic review and meta analysis. Chest 136(4): 974-982.

21. Wilson GT, Schaller FA (2008) Pulmonary embolism mimicking anteroseptal acute myocardial infarction. J Am Osteopath Assoc 108(7): 344-349.

22. Cheng TO (2009) Mechanism of ST-elevation in precordial leads V(1)-V(4) in acute pulmonary embolism. Int J Cardiol 136(3): 251-252.

23. Lin JF, Li YC, Yang PL (2009) A case of massive pulmonary embolism with ST elevation in leads V1-4. Circ J 73(6): 1157-1159.

24. Vlahakes GJ, Turley K, Hoffman JI (1981) The pathophysiology of failure in acute right ventricular hypertension: hemodynamic and biochemical correlations. Circulation 63: 87-95.

25. Adams JE, Siegel BA, Goldstein JA, Jaffe AS (1992) Elevations of CKMB following pulmonary embolism: A manifestation of occult right ventricular infarction. Chest 101(5): 1203-1206.

26. Kagiyama S, Koga T, Kaseda S, Ishihara S, Kawazoe N, et al. (2007) Transient precordial ST elevation by a spasm of the conus artery during right coronary angiography. Int J Cardiol 116(2): e57-e59.

27. Haghi D, Sueselbeck T, Papavassiliu T, Haase KK, Borggrefe M (2004) Paradoxical coronary embolism causing non-ST segment elevation myocardial infarction in a case of pulmonary embolism. Zeitschrift für Kardiologie 93(4): 824-828.

28. Johnson BI (1951) Paradoxical embolism. J Clin Pathol 4(3): 316-332.

29. Livaditis IG, Paraschos M, Dimopoulos K (2004) Massive pulmonary embolism with ST elevation in leads V1-V3 and successful thrombolysis with tenecteplase. Heart 90(7): e41.

30. Falterman TJ, Martinez JA, Daberkow D, Weiss LD (2001) Pulmonary embolism with ST segment elevation in leads V1 to V4: case report and review of the literature regarding electrocardiographic changes in acute pulmonary embolism. J Emerg Med 21(3): 255-261.

31. Homorodean C, Ober MC, Olinic M, Homorodean R, Hassoune A, et al. (2016) Takotsubo Cardiomyopathy presenting as ST-elevation myocardial infarction: wide triggering spectrum and specific echocardiographical pattern in a consecutive case series report. Med Ultrason 18(4): 475-480.

32. Susan W, Daniel JQ Giancarlo A, John WE (2012) Thrombolysis compared with heparin for the initial treatment of pulmonary embolism. Circulation 110: 744-749.

33. Stein PD, Matta F (2012) Thrombolytic therapy in unstable patients with acute pulmonary embolism: saves lives but underused. Am J Med 125(5): 465-470. 
CC This work is licensed under Creative (1) Commons Attribution 4.0 License DOI: $10.19080 /$ JOCCT.2018.09.555760
Your next submission with Juniper Publishers will reach you the below assets

- Quality Editorial service

- Swift Peer Review

- Reprints availability

- E-prints Service

- Manuscript Podcast for convenient understanding

- Global attainment for your research

- Manuscript accessibility in different formats

( Pdf, E-pub, Full Text, Audio)

- Unceasing customer service

Track the below URL for one-step submission https://juniperpublishers.com/online-submission.php 